\title{
Formation and Characterization of Nanostructured Composite Coatings Based on the TiN Phase
}

\author{
F.F. Komarov ${ }^{a}$, S.V. Konstantinov ${ }^{a}$, A.D. Pogrebnjak ${ }^{b}$, V.V. Pilko ${ }^{a}$, C. Kozak $^{c}$ \\ AND M. OPIELAK ${ }^{d}$ \\ ${ }^{a}$ Institute of the Applied Physics Problems, Kurchatov St., 7, 220108, Minsk, Belarus \\ ${ }^{b}$ Sumy University, R. Korsakov St., 2, 40007, Sumy, Ukraine \\ ${ }^{c}$ Department of Electrical Devices and High Voltages Technology, Lublin University of Technology \\ Nadbystrzycka 38D, 20-618 Lublin, Poland \\ ${ }^{d}$ Institute of Transport, Combustion Engines and Ecology, Lublin University of Technology \\ Nadbystrzycka 36, 20-618 Lublin, Poland
}

\begin{abstract}
Nanostructured thin-film coatings based on titanium nitride, doped with silicon, chromium and aluminium were the object of this study. The creation of a smooth transition layer was carried out by the changing of a supplying nitrogen flow to the vacuum chamber during the application. TiSiN, TiCrN and TiAlN coatings were deposited. The studies of the structure, elemental and phase composition of the coatings were carried out. Also, the performance of coatings was investigated. The results can be used in today's technology, such as mechanical engineering.
\end{abstract}

DOI: 10.12693/APhysPolA.125.1292

PACS: $68.55 . \mathrm{Nq}$

\section{Introduction}

One of the perspective areas of modern science and technology is creation and designing multicomponent nanostructured coatings. This is due to the fact that these coatings have unique physical and chemical properties (hardness, strength, wear resistance, heat resistance, corrosion resistance, etc.) [1-3]. Reducing the size of crystal grains of the coating allows to achieve its new fundamental properties and characteristics. Reducing the grain size of less than $15 \mathrm{~nm}$ leads to lowering the temperature of phase transition, including the melting temperature, thermal conductivity reduction, reduced elastic modulus, increase hardness, fracture toughness, decrease in wear resistance, expression of superplasticity at high temperatures and sharp increase in microhardness [4-6].

Adding a third component to the coating of titanium nitride, such as chromium, silicon, aluminium, can significantly improve the performance of the coatings. In particular the addition of aluminium to titanium nitride in a magnetron sputtering target composition allows to improve the microhardness, fracture toughness, heat resistance and the Young modulus of complex coating TiAlN [7-9]. The TiAlN and TiSiN nanocomposites consisting of TiN nanocrystals embedded in the amorphous AlN or $\mathrm{Si}_{3} \mathrm{~N}_{4}$ phases have exhibited a super hardness and excellent thermal stability, being widely applied in dry and high-speed cutting treatments [10]. The TiCrN nanocomposite is also of great interest from the point

*corresponding author; e-mail: komarovF@bsu.by of view of practical applications [11]. The goal of this research is the investigation of a correlation between the composition and structure of the coating and its mechanical and nanotribological properties.

\section{Experimental procedure}

Polished stainless steel, hard alloy, graphite, silicon and single crystal sodium chloride plates freshly split were used as substrates in all cases. Magnetron depositions were performed with the usage of $\mathrm{Ar}^{+}$ions for the $\mathrm{Ti}-\mathrm{Al}-\mathrm{N}, \mathrm{Ti}-\mathrm{Si}-\mathrm{N}$ and $\mathrm{Ti}-\mathrm{Cr}-\mathrm{N}$ systems by the sputtering of composite targets containing $\mathrm{Ti}$ as a main component and $\mathrm{Al}, \mathrm{Si}$ and $\mathrm{Cr}$ as the addition in the concentrations from 20 up to 50 at.\%.

The nitrogen flow was controlled by the optical spectra analyzer S-100. The gradient layers were formed on the stainless steel samples by the alteration of nitrogen flow steering from 0 up to $0.4 \mathrm{~Pa}$. A bias voltage of $150-200 \mathrm{~V}$ was used to improve coating properties. The vacuum chamber was evacuated to a base pressure of $5 \times 10^{-4} \mathrm{~Pa}$, and prior to deposition the substrates were cleaned by $\mathrm{Ar}^{+}$sputtering at a bias voltage of $1.5 \mathrm{keV}$ for $30 \mathrm{~min}$ to remove residual pollutants and native oxides. In order to increase the adhesion between coatings and substrates the infrared light heating of the samples up to $150-240^{\circ} \mathrm{C}$ was used.

The depth distribution of atomic species in the deposited layers, was measured by the Rutherford backscattering spectrometry (RBS). The RBS measurements were performed with $1.5 \mathrm{MeV} \mathrm{He}^{+}$. Structural properties of coatings were studied by means of transmission electron microscopy (TEM) and diffraction (TED). The TEM investigations in plan-view $(\mathrm{PV})$ geometry were carried out using a $200 \mathrm{keV}$ Hitachi $\mathrm{H}-800$. The method of X-ray 
diffraction (XRD) was applied in order to study phase composition and to determine the grain size of formed coatings [12]. To find the hardness and the elastic modules at different loads, we used the Oliver-Pharr technique [13] (Nanoindenter G200) [14]. Also microhardness was measured by means of a DuraScan 20 equipment.

\section{Results and discussion}

Figure 1 shows the RBS spectra of coatings TiAlN deposited with varying nitrogen concentration.

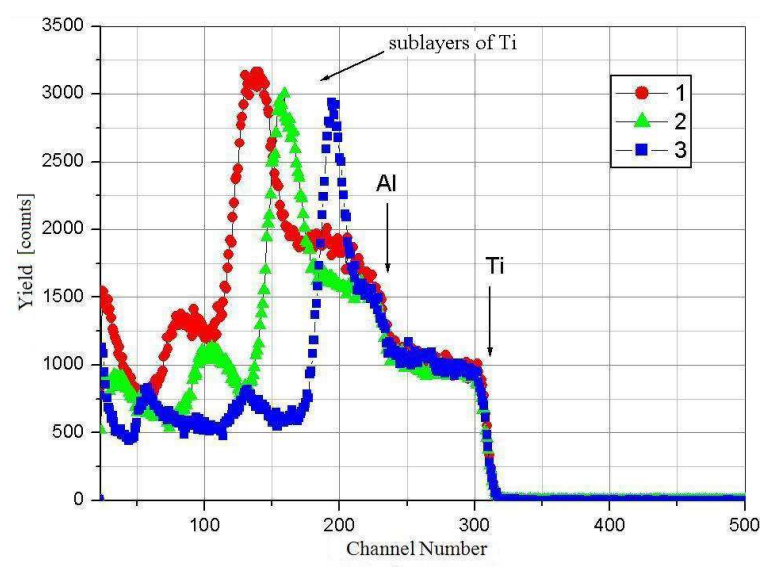

Fig. 1. RBS spectra of helium ions with an energy of 1.5 MeV from carbon substrates coated with TiAlN in different modes: 1 - with the gas drawback, 2 - with the stoichiometric concentration of nitrogen, 3 - with an excess of a reactive gas.

It was found that titanium and aluminium present in the coating in approximately equal proportions and their concentrations are homogeneous in the depth of the coating (Fig. 1). Nitrogen concentration is also practically homogeneous in the depth with a small gradient near the surface and on the boundary with the substrate. Based on the analysis of the RBS spectrum and calculated concentrations of elements it can be assumed that titanium and aluminium are in the coating in phases of nitride. Oxygen concentration in the coating is negligible compared to the concentration of nitrogen, which shows the high quality of the resulting structures and the absence of extremely harmful for operational properties of nanostructured thin-film coatings oxides and surface metal oxide films (Fig. 1). It should be noted that titanium has a substantial peak asymmetry, peaks of silicon are sufficiently uniform.

The TEM microphotograph of the TiAlN coating and electron diffraction pattern were obtained (Fig. 2) from this coating deposited by reactive magnetron sputtering.

It was observed in this image that crystallites are dispersed homogeneously in the coating. The fact of the strong broadening of diffraction peaks in the electron diffraction pattern proves nanostructuring of the TiAlN coating (Fig. 2B). Calculated crystallite size amounts to about $5 \mathrm{~nm}$.

XRD measurements reveal that peaks of titanium nitride and aluminium nitride are highly diffused and form

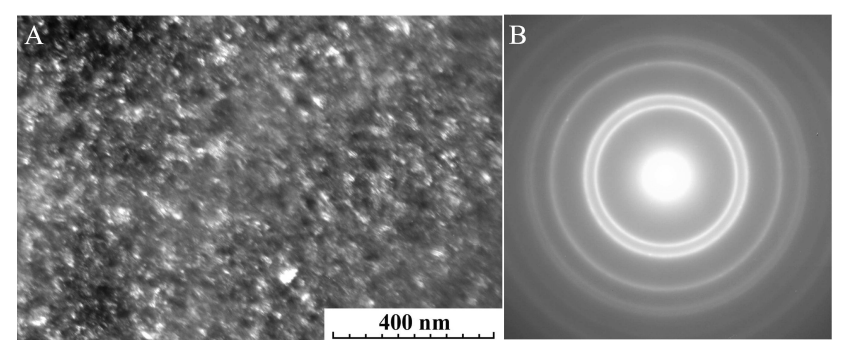

Fig. 2. Microphotograph of the coating TiAlN (A) and electron diffraction pattern from the coating TiAlN (B).

one single halo in the diffraction patterns (Figs. 3-5). As is known, both nitrides - titanium and aluminium have an isomorphic face-centered lattice and they differ only by lattice parameter and, consequently, they present unrestricted solubility in each other [1]. The atomic radius of aluminium is less than the atomic radius of titanium [1]. Assuming that the aluminium atoms replace the titanium atoms in the phase of nitride with the formation of nitride substitutional solid solution, it will shift the peak of titanium nitride in the direction of higher angles. Exactly such picture is observed in X-ray diffraction patterns obtained from the coating TiAlN (Figs. 3-5). On this basis, it is concluded that titanium nitride and aluminium nitride are in the state of the substitution solid solution, i.e. form a phase of complex nitride (Ti,Al)N (Figs. 3-5).

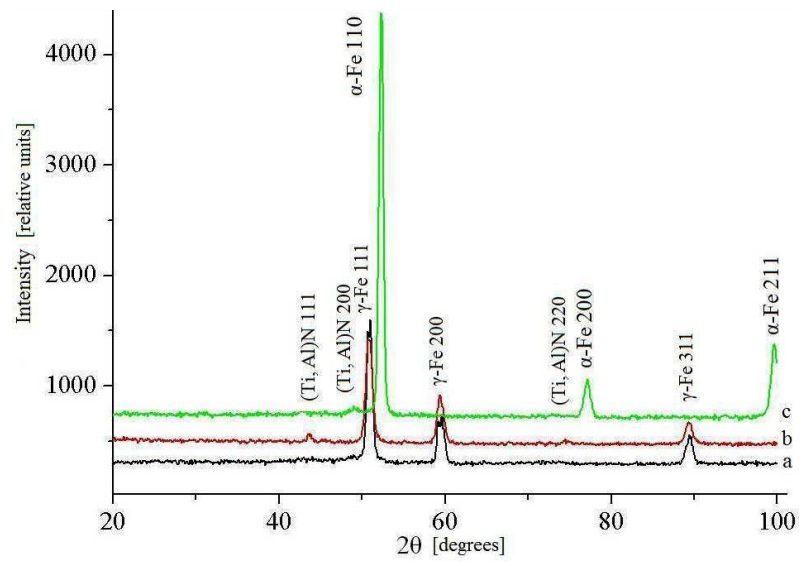

Fig. 3. X-ray diffraction patterns from $0.63 \mu \mathrm{m}$ TiAlN coating on stainless steel (a), from $0.86 \mu \mathrm{m}$ TiAlN coating on stainless steel (b), from $0.94 \mu \mathrm{m}$ coating TiAlN on $\alpha$-iron (c).

The fact of the strong broadening of peaks $(\mathrm{Ti}, \mathrm{Al}) \mathrm{N}$ with a formation of a band in the form of a halo on all diffraction patterns (a typical example is presented in Fig. 4) means the fine structure of nanocrystalline TiAlN coatings. As it was mentioned above, this fact is also proved by results obtained with TEM, where the broadening of diffraction peaks was registered, too (Fig. 2). Calculated crystallite size amounts to $4.5 \mathrm{~nm}$, that also 


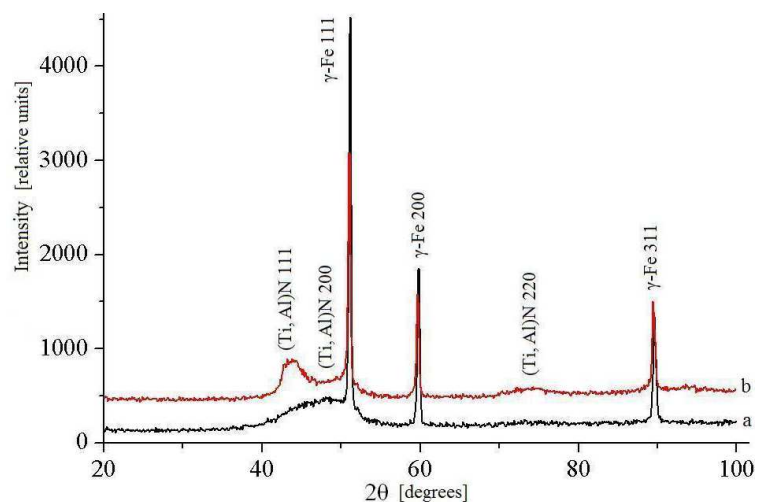

Fig. 4. X-ray diffraction patterns from the surface of TiAlN coatings deposited on stainless steel at different processing time: $55 \mathrm{~min}(\mathrm{a})$ and $75 \mathrm{~min}(\mathrm{~b})$.

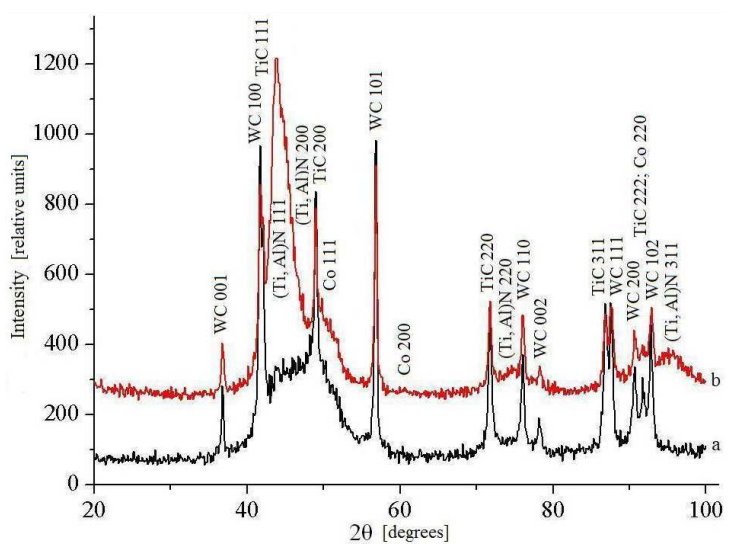

Fig. 5. X-ray diffraction patterns from TiAlN coatings on the substrate of hard alloy, the application time: $55 \min (\mathrm{a}), 75 \min (\mathrm{b})$.

accords well with results of TEM measurements.

A split of the peaks of an austenitic iron phase was observed on X-ray diffraction patterns of TiAlN coatings on stainless steel (Fig. 3a,b). This fact indicates the formation of a thin diffusion layer of a solid solution of nitrogen in the austenitic iron matrix at the substrate-coating interface.

The presence of the phase of a solid solution of nitrogen in $\gamma$-Fe matrix has been detected in the samples of stainless steel coated with TiAlN. CrN phase has not been detected in the samples coated with $\mathrm{TiCrN}$, also $\mathrm{Si}_{3} \mathrm{~N}_{4}$ phase has not been detected in the sample coated with TiSiN. This may indicate that $\mathrm{Cr}$ and $\mathrm{Si}$ atoms substitute Ti atoms in the titanium nitride phase with a formation of a solid solution with a wide range of homogeneity.

Tribological properties were investigated by nanoindentation and microindentation for more accuracy. The curves of different colors mean repeating of measurements. Exact reproducibility of the results of various measurements of nanoindentation indicates the uniformity of coatings on different areas of the samples and the homogeneity of properties of the TiAlN coatings. The presented curves of hardness indicate a high homogeneity of properties of the TiAlN coatings in the depth (Fig. 6).

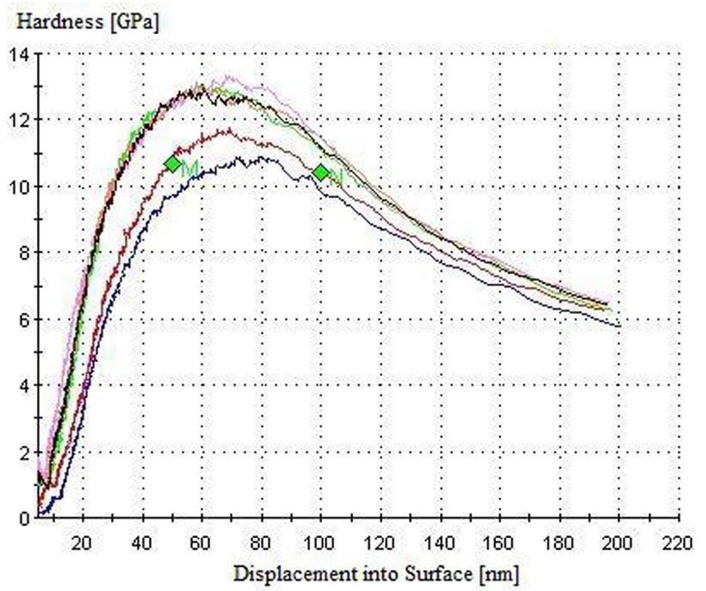

Fig. 6. Graphs of hardness of the coating TiAlN.

Also, microhardness was measured. Microhardness of TiAlN coating was identified in the range of $23-25 \mathrm{GPa}$ in the case of deposition of coatings in the mode with stoichiometric concentration and deficiency of nitrogen.

Different curves in Fig. 6 mean a repetition of measurement.

Smoothness of load-unload curves of the indentation indicates the uniformity of TiAlN coatings and their tribological properties in depth (Fig. 7).

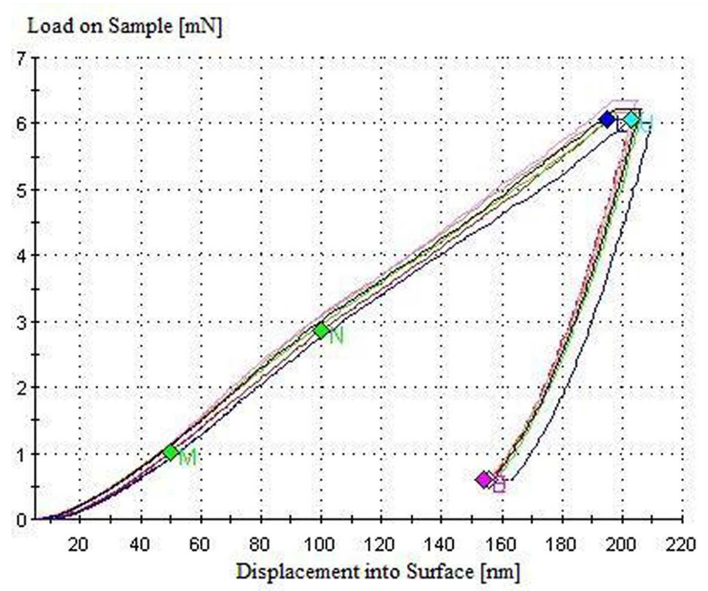

Fig. 7. Charge-discharge curves of the indenter on TiAlN coating.

Different curves in Fig. 7 also mean a repetition of measurement.

The highest modulus value was recorded on the sample of the TiAlN coating on a stainless steel substrate, the least one - on the sample of the TiAlN coating on a substrate of hard alloy (Fig. 8). Consequently, TiAlN coating better displays its elastic properties on a softer 
stainless steel substrate and imprints of the indenter recovers more after removing of the load.

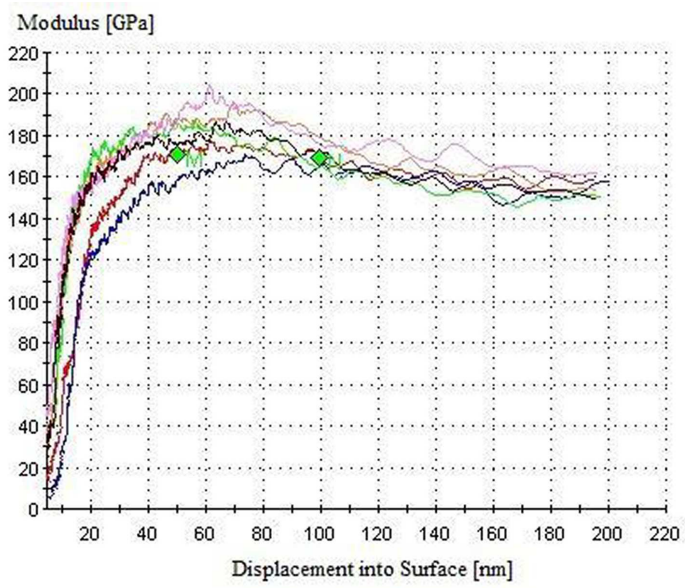

Fig. 8. Graphs of elasticity of the TiAlN coating.

Different curves in Fig. 8 mean a repetition of measurement.

The microhardness of investigated coatings increases with a decrease of the load on the indenter, as it can be seen in Fig. 6. This fact can be explained by an inevitable bursting of the thin film coating to the substrate array. Thus the hardness of the substrate itself plays an important role.

It was also established that the mode of reactive magnetron deposition with stoichiometric nitrogen concentration and with deficiency of nitrogen can provide better tribological properties, quality and larger thickness of the coating than the mode of a deposition with an excess of nitrogen.

Taking into account the RBS analysis, it can be stated that the best tribological characteristics of coatings can be achieved with close to equal concentrations of titanium and aluminium in the original target, and the final coating.

\section{Conclusions}

Doping titanium nitride coatings with aluminium, leads to a significant increase in hardness of the applied coating.

It was found that addition of aluminium in a titanium nitride coating by co-sputtering from joint target significantly changes the structure and performance of the coating.

Aluminium does not form a separate phase in the coating and exists in a state of solid solution substitution in the titanium nitride, forming a complex $(\mathrm{Ti}, \mathrm{Al}) \mathrm{N}$ nitride with face-centered cubic crystal lattice. Its lattice parameter is bigger than parameters of TiN and AlN nitrides.

Besides, a formation of a thin diffusion layer of the transition solid solution of nitrogen in the austenitic iron was revealed at the substrate-coating interface in the process of the reactive magnetron sputtering a coating onto a stainless steel substrate.
The effect of addition of $\mathrm{Al}$ on the microhardness and tribological properties of formed coatings was studied. It was found that the aluminium concentration of $50 \%$ in the sputtered target leads to the concentration of aluminium in the coating of titanium nitride in the range of $23-25 \%$. This amount of aluminium allows to achieve a significant increase in hardness of nanostructured composite coating.

It was also revealed that a significant impact on the performance of super hard thin film coatings have properties of the substrate.

According to the results of the research and identified changes in the elemental and phase compositions it can be concluded that the most promising, in order to obtain the optimum combination of properties, is the method of reactive magnetron deposition with the stoichiometric concentration and deficiency of active nitrogen gas.

The results of this research indicate a significant prospect for application of these coatings. They can be applied in various industrial fields, such as engineering: hard and superhard coatings for metal cutting tools, drills, mills, components of friction machine parts, micromechanical components, and parts.

\section{References}

[1] A. Cavaleiro, G.T. de Hosson, Nanostructure Coatings, Springer Sci. + Business Mediac, LLC, 2006, p. 750 .

[2] J. Musil, R. Daniel, P. Zeman, O. Takai, Thin Solid Films 478, 238 (2005).

[3] S. Veprek, M. Haussmann, S. Reiprich, Li Shizhi, J. Dian, Surf. Coat. Technol. 86, 394 (1996).

[4] A.L.M. Costa, A.C.C. Reis, L. Kestens, M.S. Andrade, Mater. Sci. Eng. A 406, 279 (2005).

[5] C. Jarms, H.R. Stock, P. Mayr, Surf. Coat. Technol. 206, 108 (1998).

[6] V. Chawla, R. Jayaganthan, R. Chandra, Surf. Coat. Technol. 204, 1582 (2010).

[7] S. Veprek, G.J. Maritza, V. Heijman, P. Karvankova, J. Prochazka, Thin Solid Films 476, 1 (2005).

[8] S. Veprek, G.J. Maritza, V. Heijman, Surf. Coat. Technol. 202, 5063 (2008).

[9] J. Musil, R. Daniel, J. Soldan, P. Zeman, Surf. Coat. Technol. 200, 3886 (2006).

[10] P.H. Mayrhofe, M. Stoiber, C. Mitterer, Scr. Mater. 53, 241 (2005).

[11] P. Żukowski, F.F. Komarov, Cz. Karwat, K. Kiszczak, Cz. Kozak, A.S. Kamyshan, Vacuum 83, 204 (2009).

[12] Power diffraction file data cards. Inorganic section, Sets 1-34, JCPDS, Swarthmore, Pensylvania, USA 1948-1984.

[13] V.C. Oliver, G.M. Pharr, J. Mater. Res. 7, 1564 (1992).

[14] F.F. Komarov, V.V. Pilko, A.D. Pogrebnjak, C. Karvat, K. Kiszczak, C. Kozak, Acta Phys. Pol. A 123, 800 (2013). 